\title{
Alterações do fluxo salivar total não estimulado em pacientes portadores de carcinoma espinocelular de boca e orofaringe submetidos à radioterapia por hiperfracionamento
}

Alterations of total non stimulated salivary flow in patients with squamous cell carcinoma of the mouth and oropharynx submitted to hyperfractionated radiation therapy

\author{
Maria Isabela Guebur', Abrão Rapoport' ${ }^{2}$ Laurindo Moacir Sassi ${ }^{3}$, Benedito Valdecir de Oliveira ${ }^{4}$, José Carlos Gasparin Pereira ${ }^{5}$, \\ Gyl Henrique Albrecht Ramos
}

\begin{abstract}
Resumo
A prevenção e o diagnóstico precoces são atualmente, as medidas mais eficazes de que dispomos para melhorar o prognóstico dos tumores malignos. O s tumores de boca e orofaringe são tratados com sucesso quando descobertos precocemente. A radioterapia é quase sempre um dos tratamentos de eleição para estes tumores. Q uando as neoplasias são diagnosticadas em estádios mais avançados, o tratamento muitas vezes necessita ser mais rápido para ser eficiente, e com isso os radioterapeutas lançam mão do hiperfracionamento, no qual o paciente recebe duas doses diárias de radiação, com dose diária menor por fração, mas maior ao dia, ficando em cerca de 160cG y/ 2x/dia. Q uando as glândulas salivares maiores estão presentes no campo irradiado, a xerostomia torna-se presente já na segunda semana de tratamento (1500 a 2000 cGy), alterando a saúde geral do paciente, que fica com dificuldade para se alimentar, falar e dormir. 0 objetivo deste estudo foi avaliar as alterações quantitativas do fluxo salivar total não estimulado de pacientes que se submetem ao hiperfracionamento para tratamento de carcinoma espinocelular de boca e orofaringe. Foram avaliadas as amostras de saliva de doze pacientes do $\mathrm{H}$ ospital Erasto Gaertner, de Curitiba, Paraná, pacientes esses do sexo masculino. Foram coletadas duas amostras de saliva, a primeira antes da radioterapia e a segunda, ao término do tratamento.

Como resultado, obtivemos perda salivar em $91,7 \%$ dos pacientes, com uma porcentagem de perda de fluxo salivar total de $62,9 \%$, registrada na segunda coleta. Concluindo, o hiperfracionamento ocasiona xerostomia bastante acentuada quando as glândulas salivares maiores encontram-se presentes no campo irradiado.
\end{abstract}

Palavras-chave: neoplasias orofaríngeas; neoplasias bucais; radioterapia hiperfracionada; glândulas salivares; xerostomia.

\footnotetext{
${ }^{1}$ M estre em Ciências da Saúde pelo H ospital H eliópolis, H OSPHEL-SP - Professora da D isciplina de Anatomia Humana das Faculdades Integradas "Espírita", Curitiba-PR.

${ }^{2}$ Coordenador do Curso de Pós-Graduação em Ciências da Saúde do H ospital H eliópolis, H OSPH EL - SP.

${ }^{3}$ Chefe do Serviço de Cirurgia e Traumatologia Buco-M axilo-Facial do H ospital Erasto Gaertner, Curitiba - PR; Professor do Curso de PósGraduação em Cirurgia e Traumatologia Buco-maxilo-facial da UFPR, D outorando em Ciências da SaúdeU NIFESP-SP.

${ }^{4}$ Chefe do Serviço de Cirurgia de Cabeça e Pescoço do H ospital Erasto Gaertner, Curitiba - PR.

${ }^{5}$ Chefe do Serviço de Radioterapia do H ospital Erasto Gaertner, Curitiba - PR.

${ }^{6}$ Cirurgião O ncológico/Cirurgia de Cabeça e Pescoço do H ospital Erasto Gaertner, Curitiba - PR.

Trabalho realizado no H ospital Erasto Gaertner - Curitiba - PR sendo parte da Dissertação de M estrado de M aria Isabela Guebur, H OSPH EL-SP.

Endereço para correspondência - M aria Isabela Guebur - Rua M anoel Eufrásio, 480, ap. 04 - CEP 80030-440 - Curitiba - PR

E-mail: isabelaguebur@aol.com
} 


\begin{abstract}
Prevention and early diagnosis are actually the most effective measures that we dispose to improve the prognostic of the malignant tumors. The mouth and oropharynx tumors are treated with success, when early diagnosed. The radiotherapy is almost always one of the selected treatments for these tumors. When cancer is diagnosed in advanced stages, many a time the treatment needs to be carried out swiftly to be efficient, and consequently the radiotherapists use the hyperfractionated therapy, with the patient receiving two lower doses of radiation in two sessions daily, amounting to a higher daily dosage, of about $160 \mathrm{cG} y / 2 x /$ day. When the major salivary glands are present in the radiated field, the xerostomia appears by the second week of treatment (1500 to $2000 \mathrm{cG}$ ), changing the patient's health, and causing difficulties for him to eat, speak and sleep. The objective of this study was to evaluate the quantitative alterations of the total non stimulated salivate flow of patients who underwent hyperfractionated therapy for the treatment of squamous cell carcinoma of mouth and oropharynx. Samples of twelve male patients' saliva from Erasto Gaertner Hospital in Curitiba, Paraná, Brazil, were examined. Two samples of saliva were collected from each patient, the first one before the beginning of the radiotherapy, and the second at the end of the treatment. As a result, we obtained salivary loss in $91.7 \%$ of the patients, with a percentage of total salivary flow loss of $62.9 \%$, registered in the second collection. We concluded that the hyperfractionated therapy causes a marked xerostomia when the major salivary glands are in the radiated field.
\end{abstract}

Key words: oropharyngeal neoplasms; mouth neoplasms; hyperfractionated radiotherapy; salivary glands; xerostomia.

\section{INTRODUÇÃO}

A prevenção e o diagnóstico precoces são atualmente as medidas mais eficazes de que dispomos para melhorar o prognóstico dos tumores malignos. ${ }^{1}$ Os tumores de boca e orofaringe são tratados com sucesso quando descobertos precocemente. 0 diagnóstico precoce de tumores bucais não deveria apresentar muitas dificuldades, uma vez que os grupos de maior risco são bem conhecidos e a cavidade bucal é de fácil visualização e acesso. ${ }^{2}$ Entretanto, observa-se que os pacientes não são esclarecidos e negligenciam os sintomas, e os profissionais da área da saúde não examinam rotineiramente a mucosa bucal, o que coloca o tratamento das neoplasias bucais em uma situação particularmente grave, visto que na maioria dos casos, muitas delas são diagnosticadas tardiamente. ${ }^{1}$

A faixa etária de maior prevalência do câncer bucal está entre 50-70 anos, com predileção para o gênero masculino. ${ }^{3}$

O carcinoma espinocelular (CEC) é o tumor mais comumente encontrado na região da cabeça e pescoço $(95 \%)$, e a principal causa de prognósticos desfavoráveis éo fato de estas neoplasias malignas serem diagnosticadas tardiamente, isto é, quando já se encontram em estádios avançados. ${ }^{4} \mathrm{Q}$ uando uma neoplasia é diagnosticada tardiamente, ou seja, nos estádios III e IV (UICC TNM , 2002), o tratamento é, geralmente, mais complicado e doloroso para o paciente.
A radioterapia é o tratamento não cirúrgico mais importante para o câncer. Entre $45 \%-50 \%$ dos pacientes com câncer que têm possibilidades de cura, $70 \%$ recebe a radioterapia como modalidade de tratamento. ${ }^{5}$

$Q$ uando a radioterapia é aplicada na região da cabeça e pescoço, dependendo da área irradiada, da dose de irradiação, do tempo de tratamento, da dose de distribuição e do uso associado de outras terapias, alterações reversíveis e irreversíveis podem ocorrer nos tecidos sadios local izados em áreas adjacentes à massa tumoral. ${ }^{6}$ Este é o caso das glândulas salivares maiores, que normalmente encontram-se presentes no campo irradiado, sofrendo as conseqüências da radioterapia na região de cabeça e pescoço e levando os pacientes a quadros gravíssimos de xerostomia.

Assim, avaliamos quantitativamente as perdas de fluxo salivar total não estimulado que ocorrem durante a radioterapia hiperfracionada de cabeça e pescoço quando esta é utilizada para tratamento de carcinoma espinocelular de boca e orofaringe.

\section{CASUÍSTICA E MÉTODO}

Foram analisadas as amostras de fluxo salivar total não estimulado de doze pacientes em tratamento no H ospital Erasto Gaertner, em Curitiba, Paraná, portadores de carcinoma espinocelular de boca e orofaringe, que se submeteram à radioterapia hiperfracionada na região da cabeça e pescoço e 
receberam doses de irradiação entre 5280 e 7040cGy. Todos os pacientes incluídos neste estudo assinaram um termo de consentimento antes do início do tratamento e das coletas de saliva. A média de idade dos doze pacientes foi de 54,5 anos sendo todos do sexo masculino. 0 s indivíduos foram selecionados de acordo com a chegada ao Serviço de Radioterapia deste hospital. Todos os pacientes deste estudo declaram terem sido tabagistas e/ou etilistas antes de iniciar o tratamento.

Foram excluídos desta pesquisa todos os indivíduos portadores de doenças sistêmicas ou que fizessem uso de medicamentos que pudessem alterar o fluxo salivar normal, que não tivessem todas as glândulas salivares maiores e aqueles com idade menor que 20 anos ou maior que 70 anos. Q uanto ao sítio primário da doença, os pacientes encontraram-se assim distribuídos: 1 , em base de língua e assoalho bucal, 2 em assoalho bucal e língua, 1 em retromolar, 6 em base de língua, 1 em assoalho bucal, 1 em orofaringe. (Tabela 1 )

De acordo com o método TNM (1998), de classificação de tumores malignos, encontramos: três pacientes em estádio III, oito pacientes em estádio IVa e um paciente em estádio IVb. (Tabela 1)

Q uanto à dose total de irradiação recebida pelos pacientes, tivemos um pacientecom $5280 \mathrm{cG}$ y, um paciente com 6420 cG y, três pacientes com 6480 cG y, seis pacientes com 6720 cG y e um paciente com 7040 cGy. (Tabela 1)

O s aparel hos utilizados pelo Serviço de Radioterapia do H ospital Erasto Gaertner foram de teleterapia rotacional (Theratron $780 \mathrm{C}$, Acelerador Linear Varian e Clinac 2100). As amostras de saliva foram obtidas com a realização de duas coletas principais (C 1 e C 2), realizadas antes da radioterapia $(C 1)$ e após o término do tratamento (C2). As duas coletas foram realizadas pelo método spitting, ${ }^{7}$ sendo que eram feitas cinco coletas de um minuto, para cada amostra. A amostra do primeiro minuto sempre foi desprezada, pedindo-se ao paciente para cuspi-la ou degluti-la. Em seguida, começavam as cinco coletas para a obtenção de cada amostra, conforme fora preconizado por D AWES ${ }^{8}$. Após as coletas, eram adicionados $3 \mathrm{ml}$ de água destilada a cada amostra, para que qualquer resquício de saliva que pudesse ter ficado nas bordas do funil ou da proveta (utilizados para as coletas), pudesse escoar para o fundo. Todas as amostras eram então, guardadas em um refrigerador a $60 \mathrm{C}$, por 24 horas para a eliminação de bolhas e então eram tomadas as medidas. 0 s $3 \mathrm{ml}$ de água destilada que foram acrescentados a cada amostra foram descontados na hora das medidas.

0 valor de $0,1 \mathrm{ml} / \mathrm{min}$ para o fluxo salivar não estimulado foi definido como sendo hipofunção salivar acentuada. ${ }^{9}$ A ocorrência de um fluxo salivar total não estimulado entre 0,1 e $0,2 \mathrm{ml} / \mathrm{min}$ é considerada como xerostomia, pois acumularia uma produção diária de saliva não estimulada entre 140 e $280 \mathrm{ml}$, muito aquém dos 1000 a $1500 \mathrm{ml} /$ dia considerados como produção diária normal de saliva ${ }^{10}$.

Após a aferição do volume total de cada uma das duas amostras de cada paciente, os dados obtidos foram analisados estatisticamente pelo teste de médias, onde foram comparadas as diferenças entre os valores das coletas C 1-C2.

Tabela 1 - Relação dos pacientes de acordo a idade, localização do tumor, estadiamento e dose de irradiação

\begin{tabular}{c|c|l|l|l|l}
\hline Paciente & Idade & \multicolumn{1}{|c|}{ Localização } & Estadio & Dose Total & Gl. irradiadas \\
\hline 1 & 45 & Base da língua & IVa (T3N2bM0) & 6720 cGy & Par.; SM; SL \\
2 & 63 & Base da lingua & IVa (T4N2bM0) & $6420 c G y$ & Par.; SM; SL \\
3 & 40 & Assoalho bucal & IVa (T4NOMx) & $6720 c G y$ & Par.; SM; SL \\
4 & 45 & Base da língua & IVa (T2M2cN0) & $6720 c G y$ & Par.; SM; SL \\
5 & 68 & Base da língua & III (T3NOM0) & $6720 c G y$ & Par.; SM; SL \\
6 & 43 & Assoalho bucal e língua & III (T3N1Mx) & $6480 c G y$ & Par.; SM; SL \\
7 & 55 & Assoalho bucal e língua & IVa (T4N2c M0) & $6480 c G y$ & Par.; SM; SL \\
8 & 60 & Retromolar esquerdo & III (T2NO M0) & $6720 c G y$ & Par.; SM; SL \\
9 & 66 & Base da língua & IVa (T4 N2 Mx) & $7040 c G y$ & Par.; SM; SL \\
10 & 53 & Base da língua assoalho bucal & IVb (T4 N3 M0) & $6480 c G y$ & Par.; SM; SL \\
11 & 52 & Orofaringe & IVa (T4 N2 MO) & $5280 c G y$ & Par.; SM; SL \\
12 & 64 & Base da língua & IVa (T4 Nx Mx) & $6720 c G y$ & Par.; SM; SL \\
\hline
\end{tabular}

cGy = CentiG ray; Estádio = TN M 1998; Par. -Parótidas; SM = Submandibulares; SL= Sublinguais 


\section{RESULTADOS}

Com relação aos valores obtidos na segunda coleta (C 2), realizada ao término da radioterapia, onze pacientes $(91,7 \%)$, tiveram diminuição da quantidade de saliva em relação à coleta inicial (C 1). 0 paciente 10 obteve aumento na quantidade de saliva neste período. (Tabela 2 ).

Tabela 2 - Valores das coletas de saliva total não estimulada, em mililitros $(\mathrm{ml})$, obtidos antes e após a radioterapia

\begin{tabular}{c|c|c|c}
\hline Paciente & Coleta inicial (C1) & $\begin{array}{c}\text { Segunda coleta } \\
(\mathrm{C} 2)\end{array}$ & $\begin{array}{c}\text { Diferenças entre } \\
\text { C1-C2 }\end{array}$ \\
\hline 1 & 3,4 & 0,9 & 2,5 \\
\hline 2 & 4,2 & 0,5 & 3,7 \\
\hline 3 & 2,5 & 0,2 & 2,3 \\
\hline 4 & 2,3 & 1,8 & 0,5 \\
\hline 5 & 1,7 & 0,1 & 1,6 \\
\hline 6 & 2,4 & 1,2 & 1,2 \\
\hline 7 & 3,8 & 1,8 & 2,0 \\
\hline 8 & 1,2 & 0,8 & 0,4 \\
\hline 9 & 1,9 & 0,4 & 1,5 \\
\hline 10 & 1,0 & 1,6 & $-0,6$ \\
\hline 11 & 2,5 & 1,1 & 1,4 \\
\hline 12 & 3,1 & 0,4 & 2,7 \\
\hline
\end{tabular}

$\mathrm{C} 1$ = coleta inicial; $\mathrm{C} 2$ = coleta após o término da radioterapia; diferença entre C1-C2, com sinal negativo (-), indicam que houve aumento de saliva neste período.

Q uando somados os valores obtidos nas coletas C1(antes da radioterapia) de todos os pacientes incluídos neste estudo, obtivemos $29,1 \mathrm{ml}$, e para C 2 (após a radioterapia), encontramos $10,8 \mathrm{ml}(37,1 \%$ do total encontrado antes do início do tratamento). Encontramos então, uma porcentagem de perda de fluxo salivar total entre as coletas C1-C 2 de 62,9\%, para pacientes submetidos à radioterapia hiperfracionada, e esse valor caracterizou a diminuição do fluxo salivar total não estimulado durante a radioterapia para tratamento de carcinoma espinocelular de boca e orofaringe quando as glândulas salivares maiores encontram-se presentes no campo irradiado.

A xerostomia foi encontrada em $58,3 \%$ dos pacientes, visto que estes apresentaram quantidades de saliva total entre $0,1-0,2 \mathrm{ml} / \mathrm{min}$.

O s resultados individuais das coletas, em termos de porcentagem de perda de fluxo salivar de cada paciente obtida ao final da radioterapia e as variações das medidas obtidas nas coletas C 1 (inicial) e C2 (final da radioterapia) foram calculadas em termos percentuais . (Figura 1).
Figura 1 - Gráfico representativo da variação entre as medidas obtidas na coleta inicial (C1) e coleta após a radioterapia (C2), por paciente.

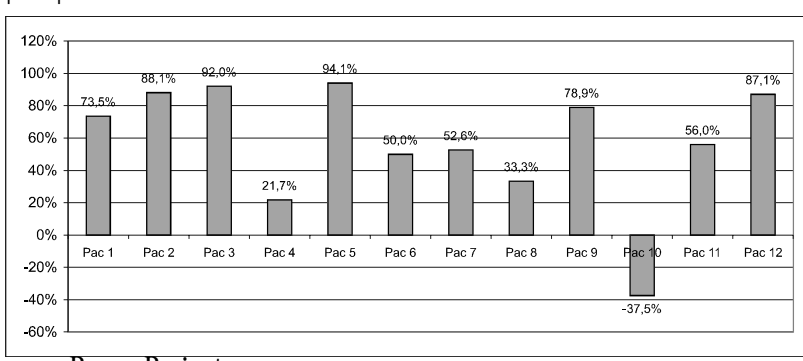

$\mathrm{Pac}=$ Paciente

\section{DISCUSSÃO}

A despeito de todos os esforços para a prevenção, 0 diagnóstico das neoplasias malignas continua sendo realizado tardiamente em muitos casos. A desinformação e a não observância dos sintomas por parte dos pacientes, e a falta de exames rotineiros das mucosas por parte dos profissionais da saúde são causas de diagnóstico tardio de tumores de boca e orofaringe, ${ }^{1}$ pois quando descobertos precocemente, são tratados com sucesso. ${ }^{2}$

Q uando uma neoplasia é diagnosticada tardiamente, necessita de um tratamento mais agressivo, o que gera efeitos deletérios mais danosos aos tecidos bucais, comprometendo muitas vezes a saúde geral do paciente e levando o radioterapeuta a recorrer a uma pausa no tratamento, para que 0 paciente possa se recuperar ${ }^{11}$.

$\mathrm{N} o$ hiperfracionamento, 0 paciente recebe duas doses diárias de irradiação e, conseqüentemente, deverá vir duas vezes ao dia ao centro de radioterapia, recebendo doses diárias bastante altas e sofrendo com os efeitos colaterais mais rapidamente do que aqueles pacientes que são portadores de neoplasias menos avançadas.

A xerostomia é o efeito deletério que primeiro acomete o paciente, e também éo mais comum, levando o paciente a sentir dificuldades em alimentar-se, poiso bolo alimentar não se forma de acordo em razão da secura bucal; em falar, pois necessita ingerir líquidos para isso; em dormir, porque a boca fica seca e as mucosas colabadas, 0 que 0 faz acordar à noite para tomar água. ${ }^{12}$

A atrofia que ocorre nos ácinos, ocasionada não só pela radioterapia hiperfracionada como também pela convencional (uma aplicação diária de irradiação), leva à diminuição do fluxo salivar ${ }^{13,14,15}$ e acarreta a perda das funções da saliva, ${ }^{16}$ deixando 0 meio bucal desprotegido e propenso a infecções secundárias, e torna a saliva escassa, espessa e pegajosa.

A diminuição do fluxo salivar não estimulado é uma constante na radioterapia de cabeça e pescoço, quando 
as glândulas salivares maiores estão presentes na área irradiada. Tal fato pôde ser observado neste estudo, em que se verificou uma perda de $62,9 \%$ entre todos os pacientes, que só não foi maior devido a um paciente (10) ter tido o seu fluxo salivar aumentado após a radioterapia, o que às vezes acontece, porém numa pequena porcentagem dos pacientes, como a que ocorreu neste estudo (8,33\%).

A xerostomia foi encontrada em $58,3 \%$ dos pacientes e registrada na segunda coleta (após a radioterapia), onde foi observado um fluxo salivar total entre $0,1-0,2 \mathrm{ml} /$ min, conforme fora determinado $0^{9,10}$ para caracterizar a xerostomia.

A xerostomia acentua, e muito, a perda de qualidade de vida, pois muda os hábitos dos pacientes, que não conseguem se alimentar sem a adição de líquidos à comida e queixam-se de não poder comer sólidos, apenas líquidos e pastosos pouco espessos, e da dificuldade de se alimentar em público.

U ma variedade de substitutos sal ivares tem sido usada há muitos anos para tentar diminuir os efeitos indesejáveis da xerostomia. Entretanto, por ser uma substância bastante complexa e desempenhar tantas funções, ela se torna difícil de ser reproduzida em laboratório. ${ }^{17}$ Além disso, a administração sistêmica do cloridrato de pilocarpina também tem sido sugerida para a estimulação da produção de saliva residual. ${ }^{18,19}$

0 uso de agentes radioprotetores como 0 aminofostine tem resultado em efeitos positivos sobre as glândulas salivares de pacientes que se submetem à radioterapia em cabeça e pescoço, principalmente nas parótidas, que são mais radiossensíveis. ${ }^{20,}{ }^{21}$.

\section{CONCLUSÕES}

A radioterapia hiperfracionada realizada em pacientes portadores de tumores avançados de boca e orofaringe ocasiona xerostomia durante a sua execução por danos aos ácinos das glândulas salivares maiores quando estas se encontram presentes no campo irradiado.

$\mathrm{H}$ á necessidade de um estudo mais aprofundado para a constatação de possíveis danos irreversíveis, causados pela radioterapia, ao parênquima das glândulas salivares maiores.

\section{REFERÊNCIAS BIBLIOGRÁFICAS}

1. Kowalski LP, N ishimoto IN . Epidemiologia do câncer de boca. In: Parise JR. 0 câncer de boca: aspectos básicose terapêuticos. São Paulo: Sarvier; 2000.

2. Clovis] $B, H$ orowitzAM, Poel D H . O ral and pharyngeal cancer: knowledgeand opinions of dentistsin British Columbia and N ova Scotia. J Can D ent Assoc 2002;68:415-20.

3. Abdo EN , Garrocho AA, Aguiar M C. Perfil do paciente portador decarcinoma epidermóide da cavidadebucal, em tratamento no $\mathrm{H}$ ospital M ário Penna em Belo H orizonte. Rev Bras C ancerol 2002;48:357-62.

4. Epstein JB, Zhang L, Rosin M. Advances in diagnosis of oral premalignant and malignant lesions. J Can D ent Assoc 2002;68:617-21.

5. Van D eW ieleC, SignoreA, Scopinaro F, Waterhouse R, $D$ ierckx RA. Imaging tumour hypoxia: wherearewe? $\mathrm{N}$ ud $M$ ed Commun 2001;22:945-7.

6. Peterson, D 'Ambrosio JA. D iagnosis and management of acuteand chronic oral complications of nonsurgical cancer therapies. D ent C lin N orth Am 1992;36:945-66.

7. N avazesh $M$, Christensen $C M$. A comparison of whole mouth resting and stimulated salivary measurement procedures. J D ent Res 1982;61:1158-62.

8. D awesC. Physiological factorsaffecting salivary flow rate, oral sugar clearence, and the sensation of dry mouth in man. J D ent Res 1987;66:648-53.

9. Tenovuo J, Lagerlöf F. Saliva. In: Thylstrup A, Fejerskov 0. Cardiologia clínica. São Paulo: EditoraSantos; 2001. p. 17-43.

10. Epstein JB, Stevenson-M ooreP, Scully C. M anagement of xerostomia. J Can D ent Assoc 1992;58:140-3.

11. Wang CC. The enigma of accelerated hyperfractionated radiation therapy for head and neck cancer. Int J Radiat O ncol Biol Phys 1988;14:209-10.

12. 0 'C onnell AC. N atural history and prevention of radiation injury. Adv D ent Res 2000;14:57-61.

13. D örr W, H endry JH . Consequential lateeffectsin normal tissues. Radiother O ncol 2001;61:223-31.

14. $M$ ateos JJ, Setoain $X$, Ferre J, Rovirosa $A, N$ aval potro $B$, $M$ artin $F$, et al. Salivary scintigraphy for assessing the protective effect of pilocarpine in head and neck irradiated tumours. N ucl M ed Commun 2001;22:651-6.

15. Warde P, $O$ 'Sullivan B, Aslanidis], Kroll BB, Lockwood G, Waldron J, et al. A phaselll placebo controlled trial of oral pilocarpinein patientsundergoing radiotherapy for head and neck cancer. IntJ Radiat O ncol Biol Phys2002;54:9-13.

16. $M$ andel ID. The functions of saliva. J D ent Res 1987;66:623-7.

17. $\mathrm{H}$ olmes S. Xerostomia: aetiology and management in cancer patients. Support C are C ancer 1998;6:348-55.

18. G arg AK, M arlo M . M anifestations and treatment of xerostomia and associated oral effects secondary to head and neck radiation therapy. JAD A 1997;128:1128-33.

19. N eidermeier W, M atthaeus C, M eyer C, Staar S, M üller RP, SchulzeH J. Radiation-induced hypossalivation and its treatment with oral pilocarpine. O ral Surg O ral M ed O ral Pathol O ral Radiol Endod 1998;86:541-9.

20. Rudat V, M eyer J, M omm F, Bendel M , H enke M , Strnad 
$V$, et al. Protectiveeffect of Aminofostine on dental heal th after radiotherapy of thehead and neck. Int J Radiat O ncol Biol Phys 2000;48:1339-43.

21. Antanadou D, Pepelassi M, Synodinou M, Puglisi M,
Throuvalas N . Prophylatic use of Aminofostine to prevent radiochemotherapy-induced mucositis and xerostomia in head-and-neck cancer. Int J Radiat O ncol Biol Phys 2002;52:739-47. 